$X_{4}=\bar{X}_{3}=-0.8090170+0.5877853 i$. These roots may be verified as correct, since they are known to be the complex fifth roots of unity, namely $X_{1}=\cos 72^{\circ}+$ $i \sin 72^{\circ}, X_{2}=\cos 288^{\circ}+i \sin 288^{\circ}, X_{3}=\cos 216^{\circ}+i \sin 216^{\circ}$, and $X_{4}=$ $\cos 144^{\circ}+i \sin 144^{\circ}$.

Convair Astronautics

San Diego, California

1. H. E. Salzer, C. H. Richards \& I. Arsham, Table for the Solution of Cubic Equations, McGraw-Hill, New York, 1958.

\title{
A Conjugate Factor Method for the Solution of a Cubic
}

\section{By D. A. Magula}

1. Introduction. This paper gives a simple method for computing the real roots of the reduced cubic equation with real coefficients,

$$
x^{3}+A x+B=0,
$$

having roots $a, b, c$. We assume $a$ to be real, since every cubic equation has at least one real root.

The method consists in factoring $B$, and setting one factor equal to $\pm \sqrt{m}$, the other $n$. For all pairs $m, n$ such that $m+n=-A, \pm \sqrt{m}$ is a root. If no such pair exists, a method of interpolation is shown.

2. Proof of Method. The reduced cubic equation (1) can be transformed, by using the relations between the roots and coefficients, into a complete cubic,

$$
p^{3}+6 A p^{2}+9 A^{2} p+4 A^{3}+27 B^{2}=0,
$$

where

$$
p=\left(-3 a^{2}-4 A\right) \text {. }
$$

Equation (2) can be written in the form:

$$
(p+A)^{2}(-p-4 A)=27 B^{2}
$$

or

$$
\frac{(p+A)}{3} \sqrt{\frac{(-p-4 A)}{3}}= \pm B
$$

Let

$$
\begin{gathered}
m=\frac{-p-4 A}{3} \text { and } n=\frac{p+A}{3} \\
n \sqrt{m}= \pm B
\end{gathered}
$$

and

$$
m+n=-A \text {. }
$$

Received September 21, 1959; in revised form, December 22, 1959. 
From (7) and (8)

$$
\sqrt{m}(-A-m)= \pm B
$$

or squaring both sides:

$$
m^{3}+2 A m^{2}+A^{2} m-B^{2}=0 .
$$

But the equation (9) is identical with the equation (1) whose roots are squared. Therefore,

$$
m=a^{2} .
$$

Thus one root of the given cubic is $\pm \sqrt{m}$. The other two roots can be found by onlving the quadratic equation resulting from the depression of the given cubic:

$$
x^{2}+a x+\left(A+a^{2}\right)=0 .
$$

3. Steps in Computation. The calculation can be carried out in the following simple operations. A model example is given: $x^{3}-7 x-6=0$.

A. Make a table with pairs of integral factors of $B$ putting one in the first column, $\pm \sqrt{m}$, and the other in the second, $n$. In each case where $m+n=-A$, the corresponding entry in the first column is a root.

\begin{tabular}{|r|r|r|r|}
\hline$\pm \sqrt{m}$ & $n$ & $m$ & $m+n$ \\
\hline 1 & -6 & 1 & -5 \\
2 & -3 & 4 & 1 \\
3 & -2 & 9 & $\frac{7}{5}$ \\
6 & -1 & 36 & $\frac{7}{7}$ \\
-1 & 6 & 1 & $\underline{-1}$ \\
-2 & 3 & 4 & \\
\hline
\end{tabular}

In this example the equation has three integer roots which appear circled in the first column.

B. If the equation has one integer root, it can be found by the above method. Then the other two can be found by solving equation (11).

C. If the equation has no integer roots, we may use this method to interpolate. For example, suppose we have the equation, $x^{3}-3=0$, in which $A=0$. We find from the corresponding table that the root is between 1 and 3 . We can then make additional entries in the table by taking values within this interval.

\begin{tabular}{|l|l|l|l|}
\hline$\pm \sqrt{m}$ & $n$ & $m$ & $m+n$ \\
\hline 1 & -3 & 1 & -2 \\
3 & -1 & 9 & +8 \\
\hline 1.4 & -2.14 & 1.96 & -.18 \\
1.5 & -2 & 2.25 & +.25 \\
\hline 1.44 & -2.08 & 2.07 & -.01 \\
1.45 & -2.07 & 2.10 & +.03 \\
\hline
\end{tabular}


Thus the real root of this equation is between 1.44 and 1.45, and this process can be repeated until the desired degree of accuracy is attained. From (B) it follows that the presence of imaginary roots in no way affects the solution of the cubic by this method.

817 West End Avenue

New York, 25, New York 